\title{
A NEW GRAVITATIONAL-WAVE SIGNATURE FROM STANDING ACCRETION SHOCK INSTABILITY IN SUPERNOVAE
}

\author{
Takami Kuroda ${ }^{1}$, Kei KotaKe ${ }^{2,3}$, and Tomoya Takiwaki ${ }^{3}$ \\ ${ }^{1}$ Department of Physics, University of Basel, Klingelbergstrasse 82, 4056 Basel, Switzerland \\ ${ }^{2}$ Department of Applied Physics, Fukuoka University, 8-19-1, Jonan, Nanakuma, Fukuoka 814-0180, Japan \\ ${ }^{3}$ Division of Theoretical Astronomy, National Astronomical Observatory of Japan, 2-21-1, Osawa, Mitaka, Tokyo 181-8588, Japan \\ Received 2016 May 30; revised 2016 July 29; accepted 2016 August 24; published 2016 September 20
}

\begin{abstract}
We present results from fully relativistic three-dimensional core-collapse supernova simulations of a non-rotating $15 M_{\odot}$ star using three different nuclear equations of state (EoSs). From our simulations covering up to $\sim 350 \mathrm{~ms}$ after bounce, we show that the development of the standing accretion shock instability (SASI) differs significantly depending on the stiffness of nuclear EoS. Generally, the SASI activity occurs more vigorously in models with softer EoS. By evaluating the gravitational-wave $(\mathrm{GW})$ emission, we find a new GW signature on top of the previously identified one, in which the typical GW frequency increases with time due to an accumulating accretion to the proto-neutron star (PNS). The newly observed quasi-periodic signal appears in the frequency range from $\sim 100$ to $200 \mathrm{~Hz}$ and persists for $\sim 150 \mathrm{~ms}$ before neutrino-driven convection dominates over the SASI. By analyzing the cycle frequency of the SASI sloshing and spiral modes as well as the mass accretion rate to the emission region, we show that the SASI frequency is correlated with the GW frequency. This is because the SASIinduced temporary perturbed mass accretion strikes the PNS surface, leading to the quasi-periodic GW emission. Our results show that the GW signal, which could be a smoking-gun signature of the SASI, is within the detection limits of LIGO, advanced Virgo, and KAGRA for Galactic events.
\end{abstract}

Key words: gravitational waves - hydrodynamics - supernovae: general

\section{INTRODUCTION}

Clarifying a correspondence between core-collapse supernova (CCSN) dynamics and the gravitational-wave (GW) signals is a time-honored attempt since the 1980s (Müller 1982). Very recently the observational horizon of GW astronomy extends far enough to allow the first detection coined by LIGO for the black hole merger event (Abbott et al. 2016). Extensive research over the decades has strengthened our confidence that $\mathrm{CCSNe}$, next to compact binary mergers, could also be one of the most promising astrophysical sources of GWs (see Ott 2009; Kotake 2013 for reviews).

Traditionally, most of the theoretical predictions have focused on the GW signals from rotational core-collapse and bounce (see, e.g., Dimmelmeier et al. 2002; Scheidegger et al. 2010; Ott et al. 2012; Kuroda et al. 2014; Yokozawa et al. 2015). In the postbounce phase, a variety of GW emission processes have been proposed, including convection inside the proto-neutron star (PNS) and in the postshock region (Burrows \& Hayes 1996), the standing accretion shock instability (SASI; Kotake et al. 2007, 2009; Marek \& Janka 2009; Murphy et al. 2009) and nonaxisymmetric instabilities (Ott et al. 2005; Scheidegger et al. 2010; Kuroda et al. 2014).

In the non-rotating core, Murphy et al. (2009) first showed in their two-dimensional (2D) models that the evolution of convective activities in the PNS surface regions can be imprinted in the GW spectrogram. The characteristic GW frequency is considered as a result of the $g$-mode oscillation excited by the downflows to the PNS (Marek et al. 2009) and by the deceleration of convection plumes hitting the surface (Murphy et al. 2009). These features have also been identified in more recent $2 \mathrm{D}$ models with the best available neutrino transport scheme (Yakunin et al. 2010; Müller et al. 2013; Yakunin et al. 2015). Furthermore, Müller et al. (2013) showed in their self-consistent 2D models that the SASI motions become generally more violent for more massive progenitors, which tends to make the GW amplitudes and frequencies higher.

Not to mention the explosion dynamics (e.g., Hanke et al. 2012; Couch 2013; Takiwaki et al. 2014; Janka et al. 2016), the GW signatures are very sensitive to the spatial dimension employed in the numerical modeling (e.g., Kotake et al. 2009; Müller et al. 2012). Due to the high numerical cost, however, only a few full three-dimensional (3D) models have been reported so far to study the postbounce GW features (without any symmetry constraints and excision of the PNS; e.g., Scheidegger et al. 2010; Ott et al. 2012; Kuroda et al. 2014). Using a prescribed boundary condition of the PNS contraction, Hanke et al. (2013) showed in their 3D models that a rapid shrinking of the PNS fosters the development of the SASI. General relativity (GR) should play a crucial role because the SASI is favored by smaller shock radii due to the short SASI's growth rate (Foglizzo et al. 2006). To have a final word on recent hot debates about the impacts of neutrino-driven convection versus the SASI on the supernova mechanism (e.g., Burrows 2013), full 3D-GR models are needed, which is also the case for clarifying the $\mathrm{GW}$ emission processes.

In this Letter, we study the GW emission from a non-rotating $15 M_{\odot}$ star by performing 3D-GR hydrodynamic simulations with an approximate neutrino transport. Using three modern nuclear equations of states (EoSs), we investigate its impacts on both the postbounce dynamics and the GW emission. Our results reveal a new GW signature where the SASI activity is imprinted. We discuss how the detectability of the signals, if detected, could provide the live broadcast that shows how the supernova shock is dancing in the core. 

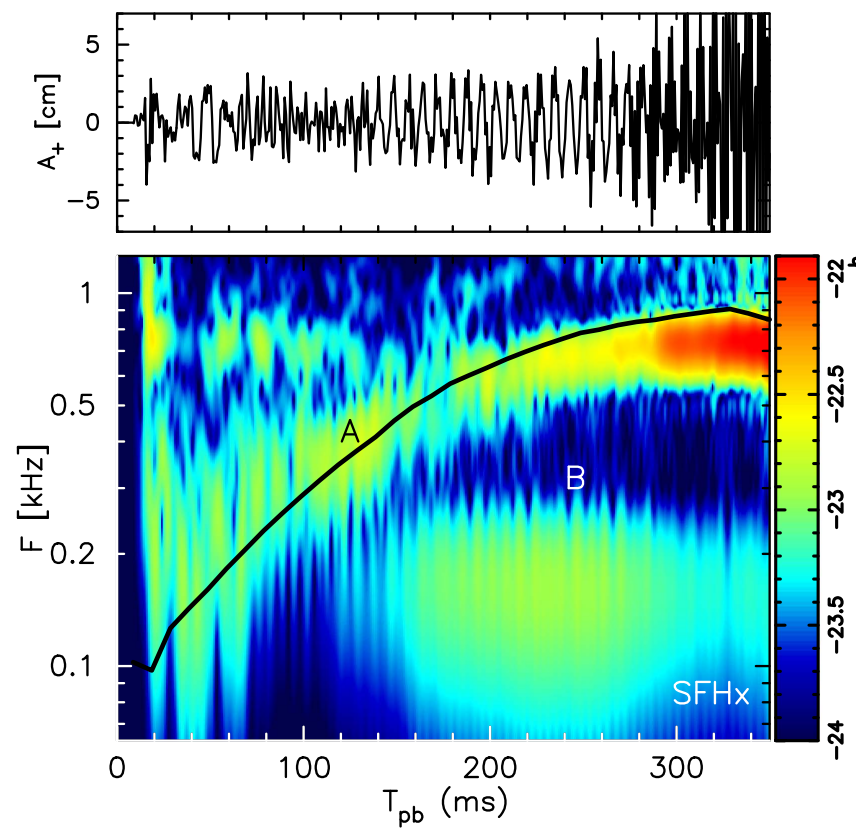
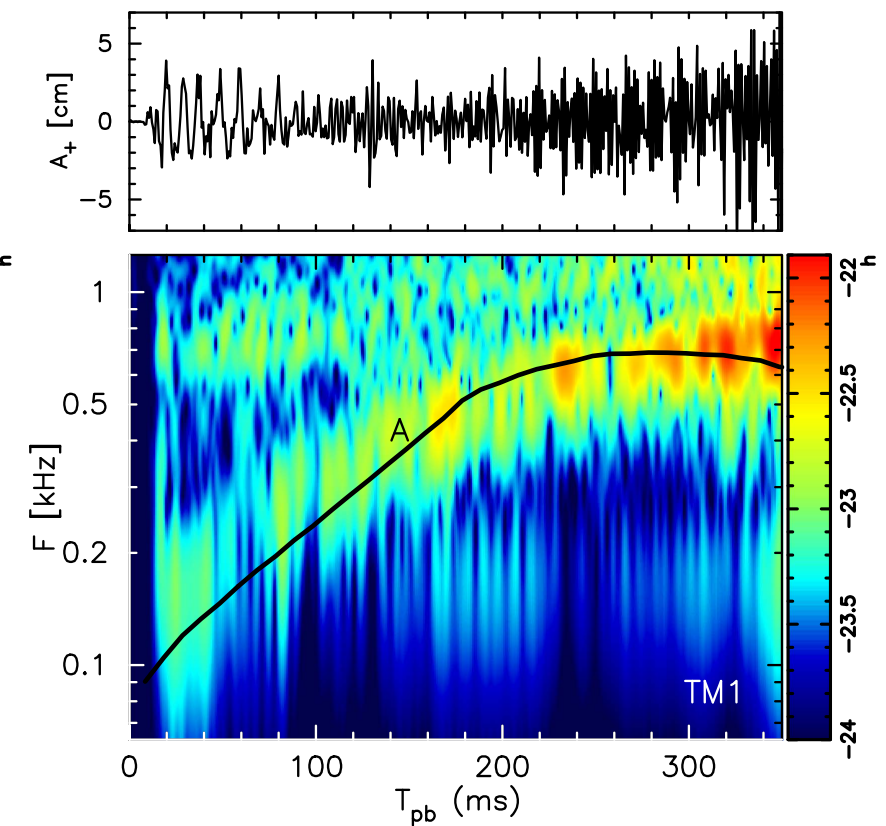

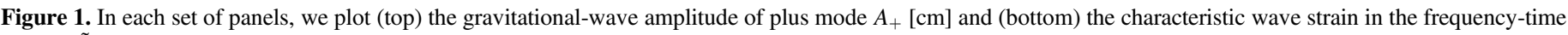

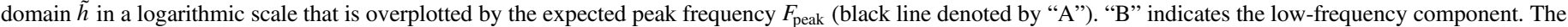

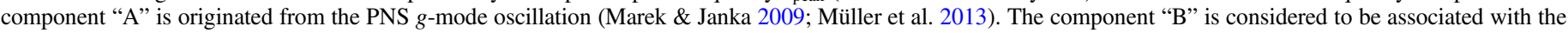

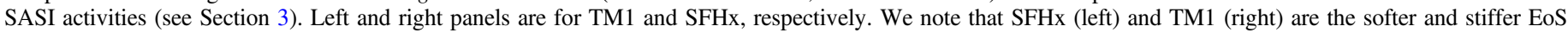
models, respectively.

\section{NUMERICAL METHODS}

In our full GR radiation-hydrodynamics simulations, we solve the evolution equations of metric, hydrodynamics, and neutrino radiation. Each of them is solved in an operatorsplitting manner, but the system evolves self-consistently as a whole satisfying the Hamiltonian and momentum constraints (Kuroda et al. 2012, 2014).

Regarding the metric evolution, we evolve the standard BSSN variables $\tilde{\gamma}_{i j}, \phi, \tilde{A}_{i j}, K$, and $\tilde{\Gamma}^{i}$ (Shibata \& Nakamura 1995; Baumgarte \& Shapiro 1999). The gauge is specified by the "1 $+\log$ " lapse and by the Gamma-driver-shift condition.

In the radiation-hydrodynamic part, the total stress-energy tensor $T_{\text {(total) }}^{\alpha \beta}$ is expressed as

$$
T_{\text {(total) }}^{\alpha \beta}=T_{\text {(fluid) }}^{\alpha \beta}+\sum_{\nu \in \nu_{e}, \bar{\nu}_{e}, \nu_{x}} T_{(\nu)}^{\alpha \beta},
$$

where $T_{\text {(fluid) }}^{\alpha \beta}$ and $T_{(\nu)}^{\alpha \beta}$ are the stress-energy tensor of fluid and the neutrino radiation field, respectively. All radiation and hydrodynamical variables are evolved in conservative ways. We consider all three flavors of neutrinos $\left(\nu_{e}, \bar{\nu}_{e}, \nu_{x}\right)$ with $\nu_{x}$ representing heavy-lepton neutrinos (i.e., $\nu_{\mu}, \nu_{\tau}$ and their antiparticles). To follow the 3D hydrodynamics up to $\lesssim 400 \mathrm{~ms}$ postbounce, we shall omit the energy dependence of the radiation in this work (see, however, Kuroda et al. 2016).

We use three EoSs based on the relativistic-mean-field theory with different nuclear interaction treatments, which are DD2 and TM1 of Hempel \& Schaffner-Bielich (2010) and SFHx of Steiner et al. (2013). For SFHx, DD2, and TM1 ${ }^{4}$, the maximum gravitational mass $M_{\max }$ and the radius of cold NS $R$ in the vertical part of the mass-radius relationship are $M_{\max }=2.13,2.42$, and $2.21 M_{\odot}$ and $R \sim 12,13$, and, 14.5

\footnotetext{
4 The symmetry energy $S$ at nuclear saturation density is $S=28.67,31.67$, and $36.95 \mathrm{MeV}$, respectively (e.g., Fischer et al. 2014).
}

$\mathrm{km}$, respectively (Fischer et al. 2014). SFHx is thus softest followed in order by DD2 and TM1. Among these three, while DD2 is consistent with nuclear experiments, such as for its symmetry energy (Lattimer \& Lim 2013), SFHx is the best-fit model with the observational mass-radius relationship. All EoSs are compatible with NS mass measurement $\sim 2.04 M_{\odot}$. (Demorest et al. 2010). Our 3D-GR models are named DD2, TM1, and SFHx, which simply reflects the EoS used.

We study a frequently used $15 M_{\odot}$ star of Woosley \& Weaver (1995). The 3D computational domain is a cubic box with $15,000 \mathrm{~km}$ width, and nested boxes with eight refinement levels are embedded. Each box contains $128^{3}$ cells, and the minimum grid size near the origin is $\Delta x=458 \mathrm{~m}$. In the vicinity of the stalled shock front $R \sim 100 \mathrm{~km}$, our resolution achieves $\Delta x \sim 1.9 \mathrm{~km}$, i.e., the effective angular resolution becomes $\sim 1^{\circ}$.

Extraction of GWs from our simulations is done by the conventional quadrupole formula in which the transverse and the trace-free gravitational field $h_{i j}$ is expressed by (Misner et al. 1973)

$$
h_{i j}(\theta, \phi)=\frac{A_{+}(\theta, \phi) e_{+}+A_{\times}(\theta, \phi) e_{\times}}{D} .
$$

In Equation (2), $A_{+/ \times}(\theta, \phi)$ represents the amplitude of orthogonally polarized wave components with emission angle $(\theta, \phi)$ dependence (Scheidegger et al. 2010; Kuroda et al. 2014), $e_{+/ \times}$denotes unit polarization tensors, and $D$ is the source distance where we set $D=10 \mathrm{kpc}$ in this Letter.

\section{RESULTS}

We start by describing the hydrodynamics at bounce. The central rest mass density $\rho_{c}$ reaches $\rho_{c}=3.69,3.75$ and 4.50 $\times 10^{14} \mathrm{~g} \mathrm{~cm}^{-3}$ for TM1, DD2, and SFHx, which is higher, as expected, for the softer EOS (e.g., Fischer et al. 2014). 

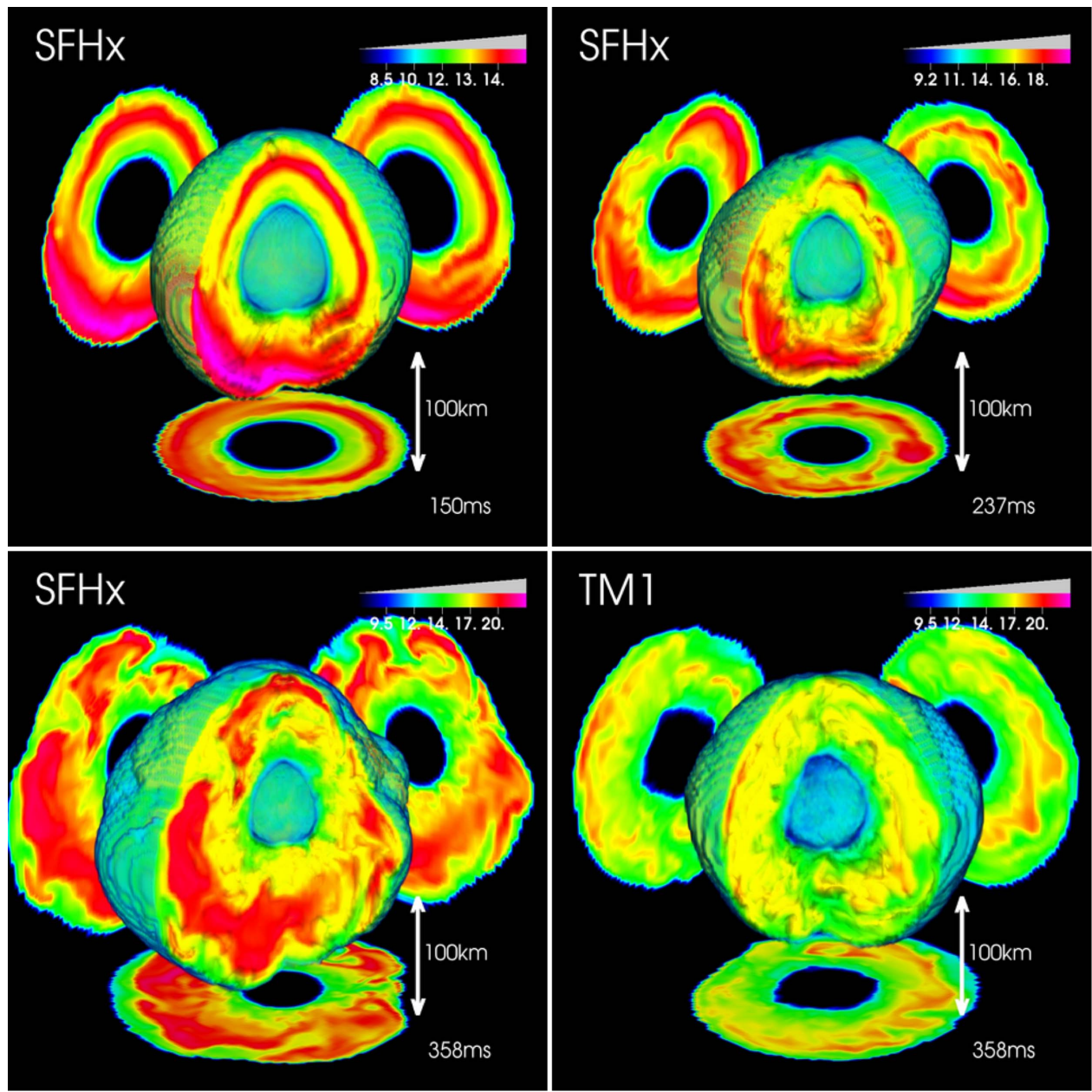

Figure 2. Snapshots of the entropy distribution $\left(k_{\mathrm{B}}\right.$ baryon $^{-1}$ ) for models SFHx and TM1 (top left, $T_{\mathrm{pb}}=150 \mathrm{~ms}$ of SFHx; top right, $T_{\mathrm{pb}}=237 \mathrm{~ms}$ of SFHx; bottom left, $T_{\mathrm{pb}}=358 \mathrm{~ms}$ of SFHx; bottom right, $T_{\mathrm{pb}}=358 \mathrm{~ms}$ of TM1). The contours on the cross sections in the $x=0$ (back right), $y=0$ (back left), and $z=0$ (bottom) planes are, respectively, projected on the sidewalls of the graphs. The $90^{\circ}$ wedge on the near side is excised to see the internal structure. Note that to see the entropy structure clearly in each dynamical phase, we change the maximum entropy in the color bar as $s_{\max }=16,20$, and $22 k_{\mathrm{B}}$ baryon ${ }^{-1}$ for $T_{\mathrm{pb}}=150,237$, and $358 \mathrm{~ms}$, respectively.

After bounce, the non-spherical matter motion develops and starts GW emission. In Figure 1, we plot time evolution of the angle-dependent $\mathrm{GW}$ amplitude (only plus mode $A_{+}(\theta, \phi)$, black line) in the top panels and the characteristic wave strain in the frequency-time domain $\tilde{h}(\theta, \phi, F)$ (see Equation (44) in Kuroda et al. 2014) in the bottom ones. Here $F$ denotes the GW frequency. We extract GWs along the north pole $(\theta, \phi)=(0,0)$. The postbounce hydrodynamics evolutions in DD2 are rather similar to TM1 and we mainly focus on the comparison between SFHx and TM1 in the following.

The GW amplitude $\left(A_{+}\right.$, top panels) shows a consistent behavior as reported in Müller et al. (2013), Ott et al. (2013), and Yakunin et al. (2015). It shows an initial low frequency and slightly larger amplitude until $T_{\mathrm{pb}} \sim 60 \mathrm{~ms}$, which is followed by a quiescent phase with a higher frequency until $T_{\mathrm{pb}} \sim 150 \mathrm{~ms}$. Afterward, the amplitude and frequency become larger with time.

From the spectrograms (bottom panels), we see a narrowband spectrum (labeled "A" in both models) that shows an increasing trend in its peak frequency. Müller et al. (2013) and Murphy et al. (2009) showed that this peak shift can be explained by properties of PNS, such as its compactness and surface temperature. By following Equation (17) in Müller et al. (2013), we overplot $F_{\text {peak }}$ in the bottom panels (black line). In both models, $F_{\text {peak }}$ indeed tracks spectral peak quite well, although there are some exceptions in the late phase of SFHx $\left(T_{\mathrm{pb}} \gtrsim 200 \mathrm{~ms}\right)$ when the other strong component appears at $100 \lesssim F \lesssim 200 \mathrm{~Hz}$ (labeled "B"). The component "A" is thus actually originated from the $g$-mode oscillation of the PNS surface.

Before going into detail to explain the origin of the lowfrequency component "B," we briefly focus on several key differences in the hydrodynamic evolution between SHFx and TM1. In Figure 2, SFHx experiences violent sloshing (top left) and spiral motions of the SASI (top right) before neutrinodriven convection dominates over the SASI (bottom left), whereas the SASI activities are less developed in TM1. For SFHx, the clear SASI motions are observed after the prompt convection phase ceases at $T_{\mathrm{pb}} \sim 50 \mathrm{~ms}$.

In Figure 3, we plot time evolutions of maximum, average, an minimum shock radii $R_{\text {shock }}$ (top, solid) and normalized mode amplitudes $\left|A_{l m}\right| \equiv\left|c_{l m}\right| /\left|c_{00}\right|$ (see Burrows et al. 2012 for $c_{l m}$ ) of spherical polar expansion of the shock surface $R_{\text {shock }}(\theta, \phi)$. For $A_{l m}$, we plot models SFHx (middle) and TM1 (bottom) with focusing a period of $120 \leqslant T_{\mathrm{pb}} \leqslant 300 \mathrm{~ms}$ that corresponds to the appearance of component "B." We also plot 

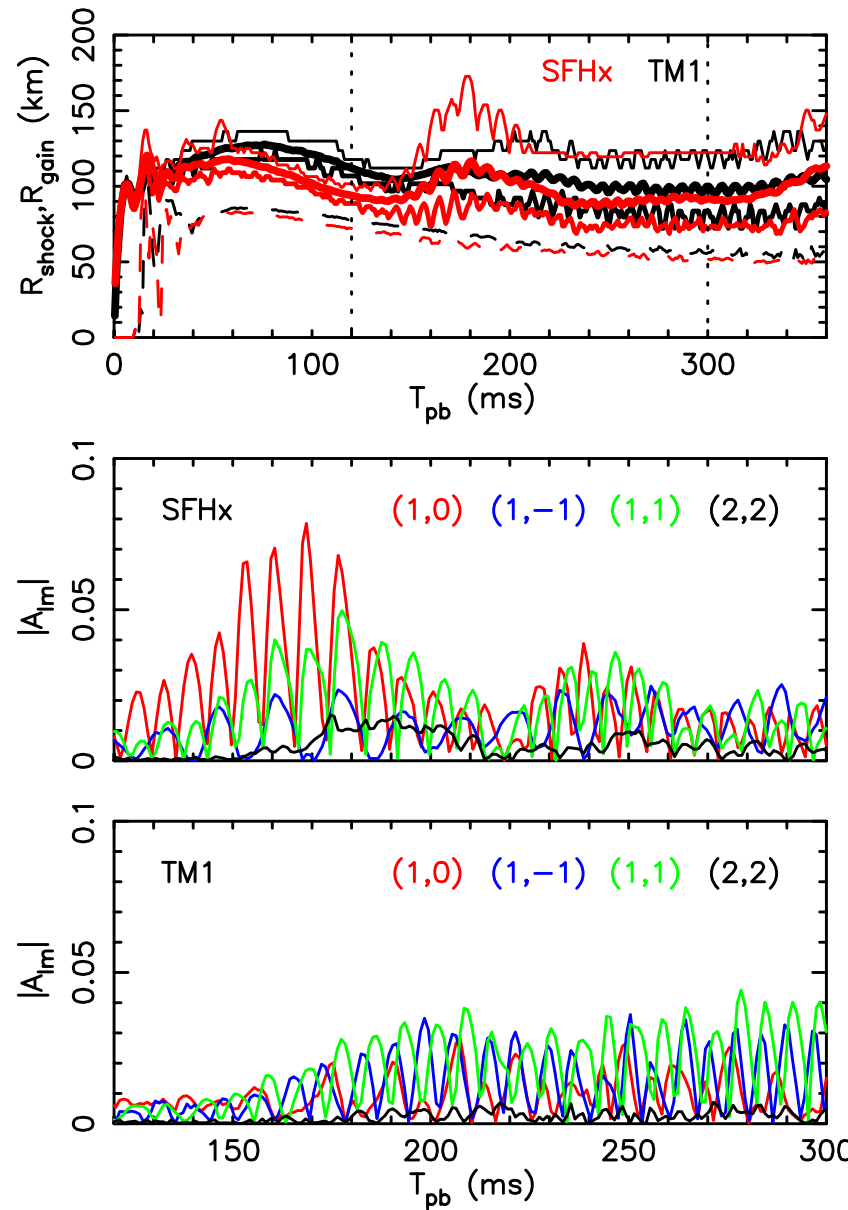

Figure 3. Top: time evolution of maximum, average, and minimum shock radii (solid) and spherically averaged gain radius (dashed) for models SFHx (red) and TM1 (black). Two vertical dotted lines represent the period when the lowfrequency component "B" appears (Figure 1). Time evolution of normalized mode amplitudes $\left|A_{l m}\right|$ for several representative modes $(l, m)$ of SFHx (middle) and TM1 (bottom). We show the period bounded by two vertical dotted lines in the top panel.

the spherically averaged gain radius $R_{\text {gain }}$ (dashed) in the top panel.

The characteristic SASI motions seen in Figure 2 are reflected in the evolution of $\left|A_{l m}\right|$. For SFHx, the most dominant mode during the first phase of the SASI $(50 \mathrm{~ms}$ $\left.\lesssim T_{\mathrm{pb}} \lesssim 150 \mathrm{~ms}\right)$ is the sloshing mode, i.e., $(l, m)=(1,0)$, which is in accord with the clear one-sided shock-heated region (top left panel of Figure 2). Regarding the EoS dependence, although we do not see any qualitative differences between the stiffest EoS model TM1 and the softest EoS model SFHx, TM1 shows less SASI development, i.e., smaller values of $\left|A_{l m}\right|$, during the SASI development phase. DD2 also shows less SASI development compared to SFHx. Such a quantitative difference can be explained by the shock radius. In the top panel of Figure 3, TM1 shows more extended shock radii until $T_{\mathrm{pb}} \sim 150 \mathrm{~ms}$. This is because, depending on the stiffness of nuclear EoS, the bounce shock can be formed at larger radius that can sometimes amount to $\sim 0.1 M_{\odot}$ difference in mass coordinates (Suwa et al. 2013; Fischer et al. 2014). Consequently, the prompt shock has to plunge into more material and stalls at smaller radius in our softest EoS model SFHx. The smaller shock radius is a favorable condition for the SASI development due to the shorter advective-acoustic cycle
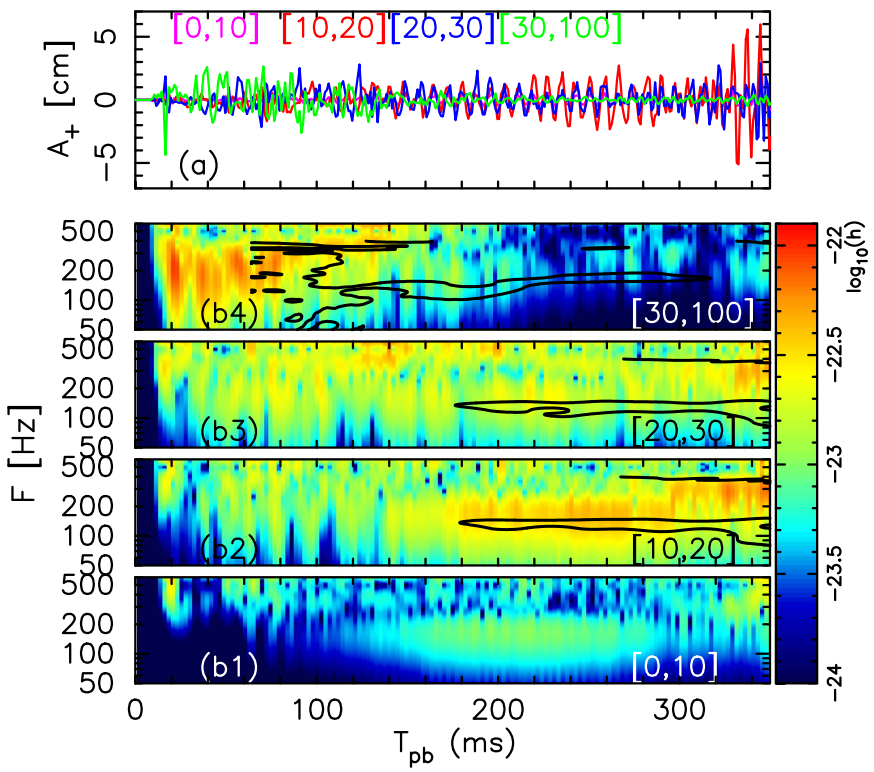

Figure 4. Rough measurement of contributions from each spherical shell to (a) the GW amplitude and (b1-4) their spectrogram $\tilde{h}$ in a logarithmic scale. We show the contributions from four spherical shells with intervals of $[0,10],[10$, 20], [20, 30], and [30, 100] km. Black contours overplotted on spectrograms for $\tilde{h}$ represent the half-maximum of spectrograms for the mass accretion rate measured at $R=17$ (b2), 23 (b3), and 48 (b4) km.

(Foglizzo 2002; Scheck et al. 2008). Initial SASI activities reach their maxima when the shock expansion occurs due to sudden drop of the mass accretion rate at $T_{\mathrm{pb}} \sim 150 \mathrm{~ms}$. Afterward, the spiral mode becomes dominant as seen in $A_{1 \pm 1}$ (see also the top right panel of Figure 2), which lasts another $\sim 150 / 200 \mathrm{~ms}$ (SFHx/TM1).

In the final phase, the core experiences neutrino-driven convection until the end of our calculation time $T_{\mathrm{pb}} \sim 350 \mathrm{~ms}$. During this phase, matters in the gain region are exposed intensively to neutrino radiations and form high entropy $\left(s_{k_{\mathrm{B}}} \sim 20\right)$ smaller-scale convection plumes (middle and bottom panels of Figure 2). Following Foglizzo et al. (2006), we check the parameter $\chi$. Although $\chi \gtrsim 3$ is expected to be satisfied for convection to develop, we find that $\chi$ stays $\sim 0.5$ until $T_{\mathrm{pb}} \lesssim 350 \mathrm{~ms}$ in both models despite the appearance of convection plumes. As already pointed out in Ott et al. (2013) and Hanke et al. (2013), this is because the initial perturbations in the gain region are already not small when the neutrino convection phase initiates. The gain radius $\left(R_{\text {gain }}\right.$ in Figure 3$)$ appears more inward in SFHx, which leads to higher entropic convection plumes compared to those in TM1 (compare the bottom two panels of Figure 2).

Now, we discuss how these hydrodynamical evolutions affect the GW emission "B" in Figure 1. By spatially decomposing the quadrupole moment of matters into several spherical shells, we roughly localize this emission at $10 \lesssim R \lesssim 20 \mathrm{~km}$ (Figure 4 ).

Before going into further discussion, we present a back-ofthe-envelope estimation of the GW amplitude as

$$
D|h| \sim 2 \epsilon M R^{2} / T_{\text {dyn }}^{2} \sim 2 \epsilon M^{2} / R \sim 2 \epsilon R^{2} \dot{M}^{2} / M
$$

where $M, R$, and $T_{\text {dyn }}$ represent the mass, size, and dynamical timescale of the system, respectively, in geometrized unit. 


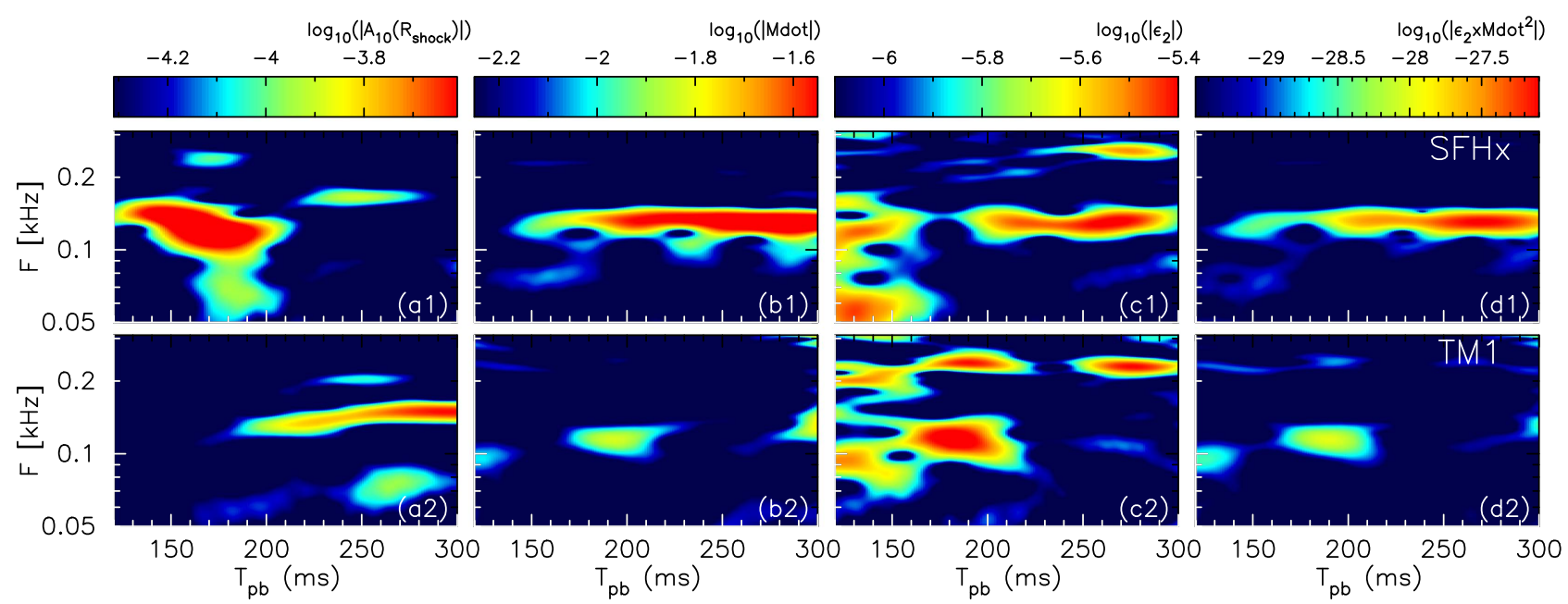

Figure 5. Spectrograms of (a) Fourier decomposed normalized mode amplitude $\left|\tilde{A}_{10}\right|$ of the shock surface for the sloshing-SASI mode; (b) the mass accretion rate $\tilde{\dot{M}}$ (with a dimension of $M_{\odot}$ ), through surface of a sphere with radius of $R=20 \mathrm{~km}$; (c) deformation of the isodensity surface $\tilde{\epsilon}_{l}$ for $l=2$ mode; and (d) a rough measurement of the GW energy spectrum that is proportional to $\sim \epsilon R^{2} \dot{M}^{2} M^{-1}$ (see the text). Top and bottom rows are for SFHx and TM1, respectively.

Here, we have used the following reasonable assumptions:

$$
T_{\mathrm{dyn}} \sim M / \dot{M}
$$

or

$$
T_{\mathrm{dyn}} \sim R / V \sim \sqrt{R^{3} / M},
$$

with $V \sim \sqrt{M / R}$ being the velocity derived by the energy conservation. From the last relation in Equation (3), we expect that significant time variation in the mass accumulation onto the PNS can potentially lead to the GW emission. In Figure 4, we superimpose the spectrogram of the mass accretion rate $\dot{M}(R)$ (the black contour at half-maximum) measured at $R=17,23$, and $48 \mathrm{~km}$ on top of the GW spectrogram. While $\dot{M}(R=48 \mathrm{~km}) \quad$ starts quasi-periodic oscillation at $F \sim 100-200 \mathrm{~Hz}$ around $T_{\mathrm{pb}} \sim 120 \mathrm{~ms}$, we find a time delay of $\sim 60 \mathrm{~ms}$ for their appearance at a deeper region $(R=17$ and $23 \mathrm{~km})$. Since the density averaged mean radial velocity between the lepton-driven $(10 \lesssim R \lesssim 20 \mathrm{~km})$ and the entropydriven $(R \gtrsim 40 \mathrm{~km})$ convection layers is $\sim 5 \times 10^{7} \mathrm{~cm} \mathrm{~s}^{-1}$, the time delay is consistent with the advection timescale over the stable layer $(20 \lesssim R \lesssim 40 \mathrm{~km}$ ). Furthermore, coincidence of time modulation in $\dot{M}(R)$ and the GW component "B" is obvious from panel (b2).

Finally, to connect the SASI activities with the GW component $\mathrm{B}$, we plot spectrograms of the normalized mode amplitude of the sloshing-SASI mode $\left|\tilde{A}_{10}\right|$, the mass accretion rate $|\tilde{\dot{M}}|$ measured at $R=17 \mathrm{~km}$, normalized quadrupole deformation of the isodensity surface $\tilde{\epsilon}_{l}$ for $l=2$, and a rough measurement of the GW energy spectrum in Figure 5. $\tilde{\epsilon}_{l}$ denotes a Fourier component of normalized mode amplitude $\epsilon_{l}$ defined by

$$
\epsilon_{l} \equiv \sqrt{\sum_{m=-l, l}\left(R_{l, m}^{14}\right)^{2}} / R_{0,0}^{14}
$$

where $R_{l, m}^{14}$ is evaluated by the spherical polar expansion of the isodensity surface $R^{14}$ extracted at $\rho=10^{14} \mathrm{~g} \mathrm{~cm}^{-1}$ as the same way as for the shock surface. Although several other modes are excited at the surface, only the leading contribution $(l=2$ mode) to the GW emission is shown in the panel. As a reference, the isodensity surface $R^{14}$ locates $\sim 13.5 \mathrm{~km}$ during $150 \lesssim T_{\mathrm{pb}} \lesssim 300 \mathrm{~ms}$ in SFHx. From the last relation in Equation (3), we plot $\log _{10}|h| \sim \log _{10} \epsilon \dot{M}^{2}+$ const. in panels (d) of Figure 5 with assuming $M=0.5 M_{\odot}$, a mass contained in $10 \lesssim R \lesssim 20 \mathrm{~km}$, and $R^{14}=13.5 \mathrm{~km}$ stays nearly constant.

During $140 \lesssim T_{\mathrm{pb}} \lesssim 180 \mathrm{~ms}$ in $\mathrm{SFHx}$, we see a strong sloshing motion that has its peak frequency at $100 \lesssim F \lesssim 200 \mathrm{~Hz}$ (a1). With some time delay ( $\sim 50 \mathrm{~ms})$ from the appearance of it, the mass accretion rate $\dot{M}$ starts showing a quasi-periodic oscillation at the same frequency range $100 \lesssim F \lesssim 200 \mathrm{~Hz}$ (b1) and it excites oscillation on the isodensity surface (c1). A combination of large $\dot{M}$ and $\epsilon_{2}$ causes us to expect GW emissions to appear in panel (d1), and it can well explain Figure 1 . During $200 \lesssim T_{\mathrm{pb}} \lesssim 300 \mathrm{~ms}, \epsilon_{2}$ stays at $\sim 3 \times 10^{-4}$ in SFHx. A rough measurement of the GW amplitude due to this deformation, $A \sim 2 \epsilon_{2} M^{2} R^{-1}$, deduces $A \sim 2 \mathrm{~cm}$, which is consistent with the actual amplitude (Figure 4).

\section{SUMMARY AND DISCUSSION}

We have presented relativistic 3D SN simulations with three different nuclear EoSs. The overall pictures of SN dynamics are qualitatively the same among all three models, although the development of the SASI differs quantitatively. The softer the $\mathrm{EoS}$ is, the more the SASI develops, since the prompt shock stalls at smaller radii. The evolution shows the first prompt convection phase, the sloshing-SASI phase, which shifts to the spiral mode and finally to the neutrino-driven convection phase.

Regarding the GWs, we have also confirmed previously reported emissions originated from the PNS surface $g$-mode oscillation (Murphy et al. 2009; Müller et al. 2013). Additionally, in the softest EoS model SFHx, in which the most vigorous SASI motion was observed, we have found another low-frequency $(100 \lesssim F \lesssim 200 \mathrm{~Hz})$ quasi-periodic emission. This emission was spatially localized at 
$10 \lesssim R \lesssim 20 \mathrm{~km}$. Through a spectrogram analysis of the SASI modes, of the mass accretion rate at $R=20 \mathrm{~km}$ and of the quadrupole mode of the central core deformation, we consider that the temporally perturbed mass accretion in association with the SASI downflows penetrates into the PNS surface and excites the oscillation at $10 \lesssim R \lesssim 20 \mathrm{~km}$, which then leads to the GW emission. Just recently, Andresen et al. (2016) have also reported a similar result that the low-frequency GW emission occurs due to the SASI. As another remarkable feature, these downflows also deform the neutrino spheres and cause time oscillation in the neutrino signals (Tamborra et al. 2013). We will report the coherency between neutrinos and GW signals originated from the SASI activity in our upcoming paper.

Finally, we briefly discuss the detectability by the next generation of GW detectors, LIGO (Harry \& LIGO Scientific Collaboration 2010) and KAGRA (Aso et al. 2013). As for the PNS surface $g$-mode oscillation, we found a dependence on the nuclear EoS. The peak frequency appears at $F=635,671$, and $681 \mathrm{~Hz}$ in TM1, DD2, and SFHx, respectively, which is in order of the stiffness of nuclear EoS. At this frequency range, the signal-to-noise ratio $(\mathrm{S} / \mathrm{N})$, a simple comparison between the energy spectra and sensitivity curves, assuming a source distance of $D=10 \mathrm{kpc}$, reaches $\sim 10$ for all the models. Regarding the SASI-origin emission "B," which is observed only in SFHx, the peak value of GW energy spectrum appears at $F=129 \mathrm{~Hz}$ and reaches almost a comparable amplitude to that from $g$-mode oscillation. The $\mathrm{S} / \mathrm{N}$ reaches a relatively high value of $\sim 50$, and because of that, both detectors have their highest sensitivity at $\sim 100-200 \mathrm{~Hz}$. From these two spectral peak values, we expect that GWs from Galactic SNe, even if their progenitors are nonrotating, are likely observable. Following Hayama et al. (2015), we plan to perform a coherent network analysis for clarifying the detectability of these signals in greater detail.

This work was supported by the European Research Council (ERC; FP7) under ERC Advanced Grant Agreement No. 321263-FISH. T.K. acknowledges valuable comments and fruitful discussions with F.-K. Thielemann and M. Hempel. Numerical computations were carried out on Cray XC30 at Center for Computational Astrophysics, National Astronomical Observatory of Japan. This study was supported by JSPS (Nos. 24103006, 24244036, 26707013, and 26870823) and by MEXT (Nos. 15H00789 and 15H01039) and JICFuS as a priority issue to be tackled by using Post " $\mathrm{K}$ " Computer.

\section{REFERENCES}

Abbott, B. P., Abbott, R., Abbott, T. D., et al. 2016, PhRvL, 116, 061102 Andresen, H., Mueller, B., Mueller, E., \& Janka, H.-T. 2016, arXiv:1607.05199 Aso, Y., Michimura, Y., Somiya, K., et al. 2013, PhRvD, 88, 043007 Baumgarte, T. W., \& Shapiro, S. L. 1999, PhRvD, 59, 024007 Burrows, A. 2013, RvMP, 85, 245

Burrows, A., Dolence, J. C., \& Murphy, J. W. 2012, ApJ, 759, 5

Burrows, A., \& Hayes, J. 1996, PhRvL, 76, 352

Couch, S. M. 2013, ApJ, 775, 35

Demorest, P. B., Pennucci, T., Ransom, S. M., Roberts, M. S. E., \& Hessels, J. W. T. 2010, Natur, 467, 1081

Dimmelmeier, H., Font, J. A., \& Müller, E. 2002, A\&A, 393, 523

Fischer, T., Hempel, M., Sagert, I., Suwa, Y., \& Schaffner-Bielich, J. 2014, EPJA, 50, 46

Foglizzo, T. 2002, A\&A, 392, 353

Foglizzo, T., Scheck, L., \& Janka, H.-T. 2006, ApJ, 652, 1436

Hanke, F., Marek, A., Müller, B., \& Janka, H.-T. 2012, ApJ, 755, 138

Hanke, F., Müller, B., Wongwathanarat, A., Marek, A., \& Janka, H.-T. 2013, ApJ, 770, 66

Harry, G. M. \& LIGO Scientific Collaboration 2010, CQGra, 27, 084006

Hayama, K., Kuroda, T., Kotake, K., \& Takiwaki, T. 2015, PhRvD, 92, 122001

Hempel, M., \& Schaffner-Bielich, J. 2010, NuPhA, 837, 210

Janka, H.-T., Melson, T., \& Summa, A. 2016, ARNPS, 66, 6

Kotake, K. 2013, CRPhy, 14, 318

Kotake, K., Iwakami, W., Ohnishi, N., \& Yamada, S. 2009, ApJL, 697, L133

Kotake, K., Ohnishi, N., \& Yamada, S. 2007, ApJ, 655, 406

Kuroda, T., Kotake, K., \& Takiwaki, T. 2012, ApJ, 755, 11

Kuroda, T., Takiwaki, T., \& Kotake, K. 2014, PhRvD, 89, 044011

Kuroda, T., Takiwaki, T., \& Kotake, K. 2016, ApJS, 222, 20

Lattimer, J. M., \& Lim, Y. 2013, ApJ, 771, 51

Marek, A., \& Janka, H.-T. 2009, ApJ, 694, 664

Marek, A., Janka, H.-T., \& Müller, E. 2009, A\&A, 496, 475

Misner, C. W., Thorne, K. S., \& Wheeler, J. A. 1973, Gravitation (San Francisco, CA: W.H. Freeman)

Müller, B., Janka, H.-T., \& Marek, A. 2013, ApJ, 766, 43

Müller, E. 1982, A\&A, 114, 53

Müller, E., Janka, H.-T., \& Wongwathanarat, A. 2012, A\&A, 537, A63

Murphy, J. W., Ott, C. D., \& Burrows, A. 2009, ApJ, 707, 1173

Ott, C. D. 2009, CQGra, 26, 063001

Ott, C. D., Abdikamalov, E., Mösta, P., et al. 2013, ApJ, 768, 115

Ott, C. D., Abdikamalov, E., O'Connor, E., et al. 2012, PhRvD, 86, 024026

Ott, C. D., Ou, S., Tohline, J. E., \& Burrows, A. 2005, ApJL, 625, L119

Scheck, L., Janka, H.-T., Foglizzo, T., \& Kifonidis, K. 2008, A\&A, 477, 931

Scheidegger, S., Käppeli, R., Whitehouse, S. C., Fischer, T., \& Liebendörfer, M. 2010, A\&A, 514, A51

Shibata, M., \& Nakamura, T. 1995, PhRvD, 52, 5428

Steiner, A. W., Hempel, M., \& Fischer, T. 2013, ApJ, 774, 17

Suwa, Y., Takiwaki, T., Kotake, K., et al. 2013, ApJ, 764, 99

Takiwaki, T., Kotake, K., \& Suwa, Y. 2014, ApJ, 786, 83

Tamborra, I., Hanke, F., Müller, B., Janka, H.-T., \& Raffelt, G. 2013, PhRvL, 111,121104

Woosley, S. E., \& Weaver, T. A. 1995, ApJS, 101, 181

Yakunin, K. N., Marronetti, P., Mezzacappa, A., et al. 2010, CQGra, 27, 194005

Yakunin, K. N., Mezzacappa, A., Marronetti, P., et al. 2015, PhRvD, 92, 084040

Yokozawa, T., Asano, M., Kayano, T., et al. 2015, ApJ, 811, 86 